\title{
La langue oudmourte dans la diaspora orientale : étude de cas
}

Udmurt in the Eastern Diaspora: Case Study

Udmurdi keel idapoolses diasporaas: juhtumiuuring

\section{Eva Toulouze}

\section{(2) OpenEdition}

\section{Journals}

Édition électronique

URL : https://journals.openedition.org/efo/1872

DOI : $10.4000 /$ efo. 1872

ISSN : 2275-1947

\section{Éditeur}

INALCO

Édition imprimée

Date de publication : 1 décembre 2013

ISBN : 978-2-343-04446-0

ISSN : 0071-2051

\section{Référence électronique}

Eva Toulouze, "La langue oudmourte dans la diaspora orientale : étude de cas », Études finnoougriennes [En ligne], 45 | 2013, mis en ligne le 05 février 2015, consulté le 08 juillet 2021. URL : http:// journals.openedition.org/efo/1872; DOI : https://doi.org/10.4000/efo.1872

Ce document a été généré automatiquement le 8 juillet 2021.

\section{(c) (7) (8)}

Études finno-ougriennes est mis à disposition selon les termes de la Licence Creative Commons Attribution - Pas d'Utilisation Commerciale 4.0 International. 


\title{
La langue oudmourte dans la diaspora orientale : étude de cas
}

\author{
Udmurt in the Eastern Diaspora: Case Study \\ Udmurdi keel idapoolses diasporaas: juhtumiuuring
}

Eva Toulouze

1 Je voudrais avec cet article rendre compte d'une expérience datant de juillet $2013^{1}$. Je décrirai sur la base de mes travaux de terrain la situation linguistique que j'ai observée dans les villages oudmourtes du nord du Bachkortostan; je m'interrogerai ensuite sur les spécificités de cette situation, sur les caractéristiques qui expliquent que l'oudmourte, pourtant langue minoritaire dans la République, s'y maintienne de façon particulièrement vivace. L'analyse des success stories a aussi son intérêt, pour réfléchir à ce qui peut - ou ne peut pas - être transposé ailleurs.

\section{Les Oudmourtes du Bachkortostan}

2 La communauté oudmourte au Bachkortostan compte un peu plus de 20000 personnes. Il s'agit d'Oudmourtes qui ont migré de leurs terres d'origine, situées dans l'actuelle république d'Oudmourtie, entre le $\mathrm{xvI}^{\mathrm{e}}$ et le $\mathrm{XIX}^{\mathrm{e}}$ siècle (Minniyakhmetova 1995, p. 331-334). Sans doute différents facteurs ont-ils motivé la migration: comme les Maris, qui ont eux aussi fui leur terre d'origine, ils ont répondu négativement à la charge fiscale, aux obligations de travail forcé (Jamurzina 2013, p. 115-118). Mais il faut reconnaître qu'aujourd'hui, les Oudmourtes dits d'outre-Kama ${ }^{2}$ ne mentionnent en général qu'un de ces facteurs: la fuite face à l'évangélisation forcée (Sadikov 2010, p. 34-35). Il faut donc croire qu'une part importante des migrations a eu lieu au cours $\mathrm{du}$ XVIII ${ }^{\mathrm{e}}$ siècle. Une fois sur place, ils ont loué ou acheté des terres. Pour prendre l'exemple de la région où a eu lieu notre terrain, c'est en 1703 que les Oudmourtes ont acquis les terres qui sont les leurs. La légende raconte qu'ils étaient six à avoir travaillé pour des Bachkirs pendant sept ans avant de pouvoir acheter la terre ; ce n'est qu'après qu'ils auraient fait venir leurs familles (Sadikov 2008, p. 10). 
3 Leur toute première activité est le travail agricole, et cela est vrai aujourd'hui comme ce l'était au $\mathrm{xx}^{\mathrm{e}}$ siècle. Avec le système soviétique se sont formés des kolkhozes, qui survivent sous une autre appellation: en effet, la forme juridique est aujourd'hui la coopérative, mais dans le langage courant, tout le monde les appelle kolkhozes. Auprès des populations voisines, avec lesquelles la coexistence est harmonieuse, les Oudmourtes ont la réputation d'être particulièrement travailleurs ${ }^{3}$ : eux-mêmes confirment cette image en faisant remarquer que dans le village, l'été, ils ne prennent jamais le temps de s'asseoir sur les bancs devant la maison, contrairement à ce qui se passe dans les villages russes et tatars, où, dès le milieu de l'après-midi, la rue devient le lieu de la socialisation. Dans le village où nous avons résidé, toute socialisation avait lieu après 20 heures, c'est-à-dire après le retour des troupeaux du pâturage et après la traite. Alors les gens se rendaient visite et restaient jusqu'à des heures tardives. Quoi qu'il en soit, les résultats économiques des kolkhozes oudmourtes sont là pour le prouver: les kolkhozes Demen et Rassvet, kolkhozes exclusivement oudmourtes, sont depuis des années en tête des résultats de production de lait, de céréales, de fourrage, ainsi que de l'élevage bovin et équin.

4 Ces kolkhozes ont été des entités solides, qui ont veillé au bon état de leurs villages : ils ont beaucoup construit au début des années 1990, par exemple les maisons de prière dans les villages d'Alga et de Vil'gurt ${ }^{4}$ (de son nom russe : Novye Tatyšly) ${ }^{5}$, mais aussi, dans ce dernier village, une cantine (qui fonctionne toujours, bien qu'elle ait été privatisée) avec quelques chambres d'hôtel à l'étage.

5 Ce n'est pas un hasard que le «Centre national et culturel des Oudmourtes du Bachkortostan ${ }^{6}$ ", organisation faisant la promotion de l'usage social de la langue oudmourte et animant la vie culturelle oudmourte de la région, se trouve dans l'école de ce même village.

6 Ainsi, grâce à la réussite économique dans l'agriculture, leur branche traditionnelle, les Oudmourtes d'outre-Kama ont pu garder de nombreuses traditions qu'on ne retrouve plus ailleurs. Parmi celles-ci, il faut évoquer les rituels qui se sont maintenus ici avec une vivacité exceptionnelle. En effet, contrairement à la majorité des Oudmourtes d'Oudmourtie, les Oudmourtes d'outre-Kama, n'ont jamais été évangélisés. Si certains d'entre eux ont été convertis, au cours de l'histoire, à l'islam, ils se sont par là même immédiatement tatarisés (Sadikov 2011). Les traditions religieuses oudmourtes ont été préservées : non sans changements ni sans évolutions, avec les mutations requises par leurs besoins internes, mais sans interférence ni pression extérieure ${ }^{7}$.

7 Bien sûr, on pouvait s'attendre à ce que la langue des pratiques religieuses soit l'oudmourte. Notre attente a effectivement été comblée, et cela m'amène à décrire la situation linguistique que nous avons rencontrée sur notre terrain. Quelques mots sur les conditions de celui-ci.

8 Dans le mois que nous avons passé dans cette région, nous avons séjourné à Bal'zjuga, un petit village de 246 habitants $^{8}$, chez une paysanne, une veuve à ce moment-là sans travail, vivant seule dans une grande maison. Nous avons partagé sa vie, connu ses voisines, ses deux filles avec leurs maris et leurs enfants, ainsi que d'autres membres de la famille. Nous étions aussi en contact avec le jeune vös'as' du village et avec sa famille (épouse, sœur, beau-frère, parents) ainsi qu'avec le vieux vös'as' du village, un patriarche prestigieux qui ne pouvait plus officier à cause de la santé de sa femme. Nous sommes également allés souvent au village de Vil'gurt (550 habitants), où se trouvaient les administrations du kolkhoze et de la commune, au village d'Alga 
(60 habitants), où a eu lieu une cérémonie, au village de Bigineevo (205 habitants), voisin immédiat de Bal'zjuga, au village d'Utar-Elga (54 habitants), où a eu lieu la Journée du village et un festival de groupes folkloriques, à Vjazovka (504 habitants), où a eu lieu un sabantuj ${ }^{10}$ de village, et bien sûr au chef-lieu du raïon, Verhnie Tatyšly.

\section{L'usage de l'oudmourte dans les villages du nord du Bachkortostan}

9 En introduction, quelques remarques sur l'usage des langues au Bachkortostan. Après qu'en octobre 1990 la République soviétique autonome de Bachkirie est devenue République du Bachkortostan, sujet de la Fédération de Russie, elle a eu un président fort, Murtaza Rahimov (président de 1993 à 2010), très engagé pour la défense de la culture bachkire et de la langue bachkire. Cette politique de bachkirisation est sensible à l'œil nu : même à Ufa, ville à dominante russe ( $48,9 \%)$, où les Bachkirs sont largement minoritaires $\left(17,1 \%{ }^{11}\right)$, toutes les enseignes sont bilingues, et ceci se retrouve dans l'ensemble du pays.

10 Mais la composition ethnique de cette République est encore plus complexe, car outre la population bachkire, plus largement présente dans le sud du pays que dans le nord ${ }^{12}$, les Tatars y sont très présents. Ainsi Russes $(36,1 \%)$, Tatars $(25,4 \%)$ et Bachkirs $(25,4 \%)$ sont-ils en proportions fort équilibrées, ce qui permet d'éviter la prédominance absolue de l'un de ces groupes.

11 La première remarque d'ensemble est que, contrairement au reste de la Russie, où l'on a un sentiment de monolinguisme envahissant, même dans des régions ethniquement diversifiées, le multilinguisme est réel et vécu dans la pratique quotidienne. Dans le bus qui nous a conduits le 24 juillet d'Ufa à Vil'gurt, nous avons bien sûr parlé russe avec le conducteur, qui nous a répondu en russe, mais celui-ci s'est adressé en tatar au reste des voyageurs, qui maîtrisaient cette langue de manière tout aussi courante, sinon plus aisée, que le russe.

12 Pour ce qui est de l'oudmourte, dans ce contexte, il est plus vivant et occupe entièrement le terrain social de la communication. En Oudmourtie, si mes amis oudmourtes de longue date parlent naturellement oudmourte avec les autres Oudmourtes, ils parlent russe tout aussi naturellement avec moi, et dans leur vie quotidienne, en dehors du foyer, utilisent plus souvent le russe que leur langue.

13 Ici, la situation est inversée. Certes, il est possible partout de parler russe et d'être compris. Mais la langue naturelle d'expression est l'oudmourte. Heureusement, nous avions avec nous Ranus Sadikov, chercheur à l'Institut Kuzeev de l'Académie des sciences de Russie, lui-même oudmourte. Sa présence a permis à nos interlocuteurs de s'exprimer librement, et sa gentillesse a fait qu'il nous a transmis les informations avec la plus grande rigueur.

14 Comme l'a fait Jack Rueter dans son article (Rueter 2013), j'énumère ci-dessous les situations dans lesquelles l'oudmourte était langue naturelle de communication, et aurait été la seule langue utilisée si nous n'avions pas été présents.

15 - À la cantine du kolkhoze Demen, à Vil'gurt. La jeune serveuse s'est adressée à Ranus en oudmourte et ils ont conversé un moment en oudmourte. C'est en oudmourte qu'elle a passé les commandes à la cuisinière et qu'elle parlait avec les jeunes manutentionnaires. 
16 - À la direction du kolkhoze. Le directeur du kolkhoze, Rinat Biktimirovič Galljamšin, a longtemps été une personnalité centrale dans le monde oudmourte. Il a une grande autorité dans toute la région. Pour des raisons de santé, il s'est retiré de la direction de la coopérative, qu'il a "léguée " à son fils Rustem (qui était chercheur en zoologie à Ufa), et il se concentre sur la propagation de la culture oudmourte. Aussi bien lui que son fils parlent oudmourte dans leur vie professionnelle quotidienne et passent au russe pour nous parler.

17 - À la direction du village. Nous avons eu quelques démarches à faire, nécessitant la participation du «maire» du village, qui a en fait sous sa compétence quatre petits villages. Nikolaj Vladimirovič Rahimjanov, enseignant d'histoire à Vil'gurt, parle oudmourte avec sa secrétaire, et quand il répond au téléphone, la plupart des conversations sont en oudmourte (nous n'en avons pas entendu d'autres, mais nous ne pouvons pas exclure qu'il parle une autre langue quand il communique par exemple avec les autorités du raïon).

18 - Avec le policier responsable de cette circonscription, Vadim, que nous avons rencontré aussi bien à la maison, quand il est venu voir qui étaient ces "étrangers ", qu'à la cérémonie d'Alga, où il connaissait tout le monde et parlait avec tout le monde en oudmourte.

19 - Dans les magasins de village. Toutes les vendeuses, aussi bien à Vil'gurt qu'à Bal'zjuga, sont oudmourtes, et elles communiquent toute la journée en oudmourte... sauf quand entre dans le magasin une personne, comme nous, clairement extérieure au village.

20 - Dans la rue, les gens se saluent en oudmourte et parlent oudmourte.

21 - Dans les conversations familiales.

22 Je développerai ce point: dans les familles, l'oudmourte est de rigueur. Même dans les familles mixtes, les époux se parlent oudmourte. Bien sûr, quand on parle de familles mixtes, il n'est pas question d'époux russes, mais tatars. Chez notre logeuse, aucun visiteur n'a spontanément parlé russe. Tous se sont d'abord adressés à elle en oudmourte, puis à nous en russe, pour aussitôt repasser à l'oudmourte.

23 C'est là un point qui me semble important à souligner : il est vrai que tous ont appris le russe et que tous le parlent, au moins à un niveau élémentaire. Les hommes sans doute mieux que les femmes, en raison du service militaire, mais tous, à l'école, ont fait leurs études en russe, avec pour la plupart des cours d'oudmourte. L'institutrice du village, à la retraite maintenant, parlait russe plutôt bien, mais c'était une exception. Car on sent bien que le russe est pour eux une langue réellement étrangère, dans laquelle ils ne pensent pas et dans laquelle ils ont du mal à exprimer des réflexions complexes et abstraites.

24 Ainsi, notre logeuse, qui n'a pas fait d'études particulières et qui a longtemps travaillé comme trayeuse au kolkhoze, avait du russe une connaissance qui lui permettait de parler avec nous de choses très simples. Mais elle avait plaisir à discuter longuement avec Ranus en oudmourte de la vie du village, des liens de parenté des gens entre eux, des pratiques religieuses.

25 Il en allait quelque peu de même avec notre informateur principal, le jeune vös'as', Fridman Habipjanov (33 ans), qui était enseignant à l'école de musique de Bigineevo. Lui et sa femme Nina, institutrice au chômage, avaient fait leurs études à Iževsk. Il avait pensé suivre un cursus technique, mais a dû y renoncer, car il avait du mal avec le russe, et il s'est rabattu sur des études musicales. Fridman était plus disert en 
oudmourte qu'en russe. Sa femme et sa sœur avaient l'air d'être plus à l'aise que lui pour converser en russe. Les chiffres illustrent notre constatation : d'après les données de 2010, sur 1000 Oudmourtes, 896 considèrent l'oudmourte comme leur langue maternelle et 86 nomment le russe dans cette position. Cette proportion est la plus élevée de tous les peuples du Bachkortostan (sauf les Russes). Et seuls les Bachkirs sont moins russifiés (77) que les Oudmourtes ${ }^{13}$.

Cela a d'ailleurs eu une incidence sur nos activités de terrain. Nous nous sentions handicapés, et nous n'avions aucune envie d'obliger nos interlocuteurs, par nos questions, à s'exprimer dans une langue qui leur posait problème. Nous avons préféré travailler par l'intermédiaire de Ranus, limitant nos conversations directes à des thèmes quotidiens. Dans notre longue expérience de terrain en Russie - dans l'ensemble des régions finno-ougriennes de Russie centrale, en Sibérie Occidentale, en Extrême-Orient pour Liivo-, nous n'avions jamais été confrontés à cette situation. Ailleurs, faire du terrain en russe était confortable, l'utilisation de la langue vernaculaire aurait plutôt limité les possibilités de conversation. Ici, c'était le contraire. Je vais énumérer quelques-unes des raisons de la vitalité de l'oudmourte dans cette région (comme dans toute la région d'outre-Kama : dans le village de Kirga, où a eu lieu en 2013 la cérémonie religieuse de tous les Oudmourtes d'outre-Kama, le responsable de l'administration est un Tatar, qui n'en parle pas moins parfaitement l'oudmourte, comme il l'a prouvé dans un discours improvisé après la cérémonie).

\section{Une zone homogène}

La première remarque que nous sommes amenés à faire est que l'habitat ici est tellement compact que, partout, on est entouré d'Oudmourtes. Dans cette République multinationale, on peut parler d'un milieu totalement homogène ethniquement. Les chiffres au sein même du raïon de Tatyšly parlent d'eux-mêmes :

Juda, 196 habitants, $95 \%$

Starokal'mijarovo, 438 habitants, $98 \%$

Petropavlovka, 312 habitants, $99 \%$

Vjazovka, 613 habitants, $87 \%$

Aribaš, 308 habitants, $91 \%$

Nižnebaltačevo, 775 habitants, $89 \%$

Alga, 66 habitants, $95 \%$

Bigineevo, 198 habitants, $95 \%$

Verhnebaltačevo, 185 habitants, $98 \%$

Dubovka, 35 habitants, $94 \%$

Ivanovka, 144 habitants, $98 \%$

Staryj Kyzyl-Jar, 211 habitants, $97 \%$

Tanypovka, 196 habitants, $99 \%$

Utar-Elga, 59 habitants, $95 \%$

Vil'gurt, 611 habitants, $82 \%$

Majsk, 96 habitants, $97 \%$

Malaj Bal'zjuga, 291 habitants, $99 \%$

Urazgyl'dy, 490 habitants, $93 \%$

Ces chiffres illustrent bien le caractère homogène de la population. Clairement, les quelques pourcents de non-Oudmourtes représentent les époux, qui, la plupart du 
temps, sont parfaitement intégrés à la population dominante. L'homogénéité du milieu est donc la première condition qui explique la vitalité de l'oudmourte dans ces contrées.

31 Cela explique beaucoup, mais pas tout. Certes, le besoin et l'occasion de parler une autre langue ne se font pas sentir : le contexte est exclusivement oudmourte, les gens vivent conformément à leur héritage indiscuté (non sans intégrer, bien sûr, les innovations qui leur conviennent) et l'écosystème est harmonieux. Aucune industrie n'est venue envahir cette zone exclusivement agricole. En même temps, le fait que la population soit toute de la même nationalité ne présume pas en soi, en Russie, de l'utilisation de la langue vernaculaire. En Oudmourtie, il n'est pas rare que des Oudmourtes se parlent entre eux en russe. Au Bachkortostan, les anciens étudiants ont coutume de dire qu'à Iževsk, si on entend parler oudmourte dans la rue à proximité de l'université, on peut être sûr que ce sont des étudiants originaires d'outre-Kama. La réalité de cette affirmation est pour le moins discutable, surtout aujourd'hui, mais elle a son intérêt en tant que stéréotype.

\section{Une communauté traditionnelle}

Sans doute tout aussi essentiel est le fait que les Oudmourtes de cette région soient tous engagés dans des activités liées à la vie communautaire du village. La toute première est bien sûr l'activité agricole.

Celle-ci a deux faces. D'abord, l'emploi est fourni par le kolkhoze. Mais celui-ci occupe moins le terrain qu'avant. Sur plusieurs villages regroupant un petit millier d'habitants, le kolkhoze n'emploie qu'une centaine de travailleurs (information orale de N. V. Rahimjanov).

En réalité, il y aurait, semble-t-il, du travail pour plus de personnes. Le président du kolkhoze, Rustem Rinatovič, le proclame haut et fort. Mais, dit-il, les jeunes ne veulent pas travailler dans l'agriculture. Ils préfèrent passer leurs journées sur leur ordinateur, à chatter sur Vkontakte (le Facebook russe). Il faut reconnaître aussi - mais cela, il ne le précise pas - que les salaires sont minimes et n'attirent personne. Mais l'activité agricole ne concerne pas que le travail salarié. En réalité, tout habitant du village quel que soit son statut, est un paysan. En effet, tous, trayeuses, vendeuses, enseignants, directeur d'école, brigadier, chauffeur, ont leur hozjajstvo, c'est-à-dire leur ferme à faire marcher. Tous ont un lopin de terre de quelques hectares sur lequel ils plantent, souvent des pommes de terre; un potager pour les légumes frais ; des bêtes, surtout des vaches et des moutons. Chacun a des moutons, presque tout le monde a des vaches. Notre logeuse avait sept moutons, mais un seul veau, parce qu'étant seule, elle n'avait pas suffisamment de foin pour entretenir une vache l'hiver - le veau était destiné à être vendu à l'automne. Vaches et moutons sont emmenés collectivement l'été au pâturage, et ils y passent toute la journée, sous la surveillance d'un ou deux habitants du village. C'est le village lui-même qui répartit les tours de travail suivant le nombre de bêtes de chacun, et chaque habitant prend la responsabilité de l'ensemble du cheptel.

Par ailleurs, les cochons ne sortent pas, ils sont nourris à l'étable. En revanche, les rues des villages oudmourtes, ici comme en Oudmourtie, sont caractérisées par la présence de troupeaux d'oies qui se gèrent tout seuls, sortent de la maison, vont à l'étang, picorent ce qu'ils trouvent et rentrent le soir, tout seuls. Certains ont aussi des canards et, bien sûr, des poules. Il n'est pas difficile d'identifier de loin les endroits où se trouve 
un village : en général des autours ou des corbeaux tournent en rond sur ces localités, pour le cas où ils arriveraient à saisir un poussin.

Tout ceci demande beaucoup de travail, mais c'est un travail pour soi, en dehors des activités professionnelles. Dans quelle mesure cette activité partagée par tous contribue-t-elle à maintenir la langue en activité ? C'est que ce qui se maintient, avant tout, c'est une communauté. Celle-ci est cohérente non seulement par l'origine de ses locuteurs, mais aussi par son mode de fonctionnement en tant que communauté.

Un bon exemple de ce vécu communautaire est à chercher dans les pratiques rituelles. Le village est l'unité première. Au mois de juin, le cycle rituel commence par le gurt vös', la prière du village; ensuite, deux groupes d'une dizaine de villages organisent des cérémonies communes. Une partie de la population se rassemble pour la phase finale, celle de la consommation du gruau rituel. Les gens s'installent en cercles familiaux, en général par terre, et mangent dans une écuelle posée au milieu du cercle. Mais la participation directe n'est pas la seule expression de cohérence communautaire. Avant la cérémonie, dans tous les villages concernés, on fait du porte-à-porte pour collecter du beurre et des céréales. Après la cérémonie, le vös'as' rapporte dans son village du gruau sacrificiel, qu'il distribue aux habitants, de sorte que le rituel se tient avec la participation de tous, et que tous participent du résultat matériel de la cérémonie en consommant le gruau (kaša) rituel.

\section{Un territoire non russe}

L'une des raisons essentielles du maintien organique d'une communauté oudmourte, je dirais même d'une société oudmourte, tient sans doute à l'environnement plus général. Je l'ai évoqué au début de cet article : le Bachkortostan est une région caractérisée par un équilibre non pas binaire, mais tripartite des ethnies. Je n'entrerai pas dans le détail des relations tataro-bachkires, compliquées à souhait. Le point que je tiens à souligner, c'est que les Russes, en tant que population, ne sont pas dominants dans leur présence au quotidien, alors qu'ils le sont de manière écrasante au niveau de l'État central. Dans le raïon de Tatyšly, ils ne représentent que $14,9 \%$ de la population, manifestement concentrée dans le chef-lieu. De manière générale, dans la République, ils sont confrontés à une forte minorité tataro-bachkire, c'est-à-dire à tradition musulmane et avec une forte conscience ethnique.

Cette présence russe plus feutrée qu'ailleurs se sent dans la discrétion de l'Église orthodoxe russe. Par exemple, toujours dans le raïon de Tatyšly, il n'y a pas une seule église orthodoxe. La première religion reconnue au niveau de l'État est l'islam. Au sabantuj du raïon, c'est le mullah qui a fait une lecture coranique en ouverture des festivités.

Ce qui me paraît essentiel, c'est ce qu'Arzamazov (p. 17) appelle « l'aile protectrice » ou encore «l'ombre salvatrice tataro-bachire». C'est du fait de leur présence et de leur puissance, que la présence russe n'a pas réussi à mettre en cause, que les Russes n'ont pas pénétré plus en profondeur ces contrées, permettant aux Oudmourtes de vivre leur vie sans interférences.

41 Les Russes, en effet, dans leur expansion impériale, ont toujours pratiqué et continuent à pratiquer une stratégie pragmatique: ils ne se fixent pas de buts irréalistes, mais procèdent progressivement, en abordant les tâches les unes après les autres. Nous en avons plusieurs exemples dans l'histoire : quand la conquête de Kazan', en 1552, a mis 
les Moscovites à la tête d'un empire multiethnique et multiculturel, la pénétration russe s'est faite aussi par étapes. L'Église orthodoxe a commencé par installer des monastères et construire des églises dans les villes, afin de desservir la population russe, mais surtout de construire son assise. Ce n'est que plus tard qu'elle a entrepris de convertir les populations au christianisme. Et encore, pas les populations musulmanes, ou du moins pas prioritairement: les populations animistes étaient beaucoup accessibles, car elles n'avaient aucun a priori de principe contre le christianisme, ni aucun dogme à lui opposer.

Autre exemple: les bolcheviks, dans leur quête d'alliés pendant la guerre civile, ont tenté de rassembler tous ceux qui avaient des raisons de ne pas être satisfaits de l'Ancien Régime. Donc les nationalités. C'est avec leur aide qu'ils ont réussi à asseoir leur pouvoir ; ensuite seulement, ils ont abattu leurs anciens alliés, et ont entrepris de réaliser leur projet - qu'ils n'auraient guère pu mettre en œuvre d'emblée.

Dans cette stratégie progressive destinée à asseoir un pouvoir et à faire siennes (cf. le terme osvoenie) des régions toujours nouvelles, les aires finno-ougriennes ont été une proie facile, car elles étaient à la fois imbues d'une vision du monde tolérante, moins aguerries que les dogmatiques de tout bord, relativement peu belliqueuses (à l'exception des Maris), et dépourvues de projets politiques ambitieux. En Oudmourtie, cette stratégie a eu un succès total : aussi bien dans la perspective de la christianisation, pratiquement achevée quand, en 1767, Catherine II lança l'édit de tolérance religieuse, qu'à l'époque bolchevique.

Mais outre les faits historiques, qui en soi expliquent la pénétration russe puis soviétique dans les aires périphériques, les Russes transportaient avec eux une mentalité de colonisateurs. Ils se considéraient hérauts de "culture »: les peuples qu'ils rencontraient sur leur chemin, avec leurs formes particulières de culture, leur étaient inférieurs sur l'échelle de la civilisation ${ }^{14}$. Or ceci a eu des conséquences sur l'univers mental des autochtones eux-mêmes. Pas sur tous: cette idée, pourtant existante, n'a pas suffi aux Estoniens pour se percevoir comme inférieurs (cf. Miropiev 1908, p. 184-185), ni aux Tatars, héritiers d'un État lui aussi impérial. Mais en tout cas, sur beaucoup de Finno-ougriens, cette vision du colonisateur a eu des effets destructeurs, car elle a été intériorisée par les colonisés, qui ont eu tendance à se considérer eux-mêmes comme un peuple de seconde zone. Cette perception s'étend à la langue, perçue par les uns comme étant "une langue de sauvages", et par leurs porteurs comme étant une marque de leur infériorité.

Cette vision manichéenne, caricaturale, était encore largement répandue quand j'ai commencé mes voyages en Oudmourtie en $1994^{15}$. Elle était combattue, certes, par les militants du mouvement national, qui tenaient à affirmer leur dignité, mais elle était largement présente dans le corps social. Depuis, les choses ont évolué et les attitudes sont en train de changer. Les jeunes générations qui se disent ou se sentent russes découvrent, dans bien des régions, qu'elles y ont tout de même des racines; elles découvrent avec intérêt la culture autochtone, et les limites de l'identité, bien marquées au $\mathrm{xx}^{\mathrm{e}}$ siècle, s'estompent. Qui est oudmourte? Seulement celui qui parle oudmourte? Ou bien celui qui ne parle plus oudmourte, mais qui a des racines oudmourtes, et qui n'y est pas indifférent, a-t-il lui aussi le droit de se dire tel ? Ce sont des questions complexes, qui caractérisent le début du xxi ${ }^{\mathrm{e}}$ siècle.

Mais si le mépris ouvertement exprimé il y a encore quelques décennies est aujourd'hui moins apparent, les dégâts qu'il a produits dans les mentalités n'en a pas pour autant 
disparu. Comme le dit Galina Nikitina, qui est bien placée pour le savoir : «Tant que l'élite ignorera la langue locale, il y aura très peu de chances que la langue dépasse le stade fonctionnel familial et domestique" (Nikitina 2013, p. 96). Effectivement, les intellectuels de sa génération ont élevé leurs enfants en russe. La société oudmourte doit panser ces plaies, anciennes et longuement entretenues par le contact permanent avec le peuple dominant.

D'ailleurs, dans la diaspora, il est frappant de constater des attitudes similaires : parmi les Oudmourtes de la région de Perm', dans le raïon de Kueda, le désintérêt pour la " cause » oudmourte est considérable (Nikitina 2011, p. 6). « À quoi sert l'oudmourte? " est, semble-t-il, une phrase souvent entendue dans ce contexte (Šeda-Zorina 2004).

Or, comme nous indique cette même source, au Bachkortostan, ce n'est guère le cas. Les $50 \%$ de Russes qui y vivent n'ont jamais été en position suffisamment dominante pour imposer de manière exclusive leur point de vue, leur manière de percevoir le monde. Que le christianisme n'ait pas pénétré dans les campagnes du nord du Bachkortostan relève de ce même phénomène. Il y a eu, certes, quelques tentatives à la fin du $\mathrm{XIX}^{\mathrm{e}}$ siècle, mais les Oudmourtes, avec l'expérience historique éminemment négative qui les avait amenés à quitter leur pays d'origine, ont chassé les intrus (Sadikov, Minnijahmetova 2012). Aujourd'hui encore, l'Église orthodoxe continue de lutter contre les religions locales, les considérant comme des religions arriérées (cf. Toulouze 2012, p. 262-263). Or les musulmans n'ont pas fait preuve à l'égard des pratiques cultuelles oudmourtes de la même arrogance.

Le paysan oudmourte du raïon de Tatyšly n'a jamais été exposé au mépris et à la condescendance. Il n'a pas été habitué à être placé au bas d'une échelle qui n'a pour lui aucune signification. Donc, son opinion de lui-même n'est pas entachée de critères exogènes teintés de racisme; il se comporte de manière saine et perçoit sa langue comme son outil d'expression, celui qui lui est propre et qui lui permet de s'exprimer de la manière la plus précise et la plus intime.

\section{Conclusion}

Que peut-on tirer comme enseignement de cette étude de cas réjouissante?

L'état de maintien et de vivacité de la langue oudmourte est lié à un certain nombre de conditions qu'il est sans doute difficile de reproduire totalement. Pourtant, l'homogénéité oudmourte du territoire existe dans certaines régions d'Oudmourtie : les raïons d'Alnaši, de Šarkan et de Malaja Purga (Oudmourtie du Sud) présentent une dominante oudmourte respectivement de $81,7 \%$ et, pour les deux derniers, de $83 \%$; ceux de Glazov et Debësy (Oudmourtie du Nord) n'en sont pas loin avec $79 \%^{16}$. Très probablement, dans ces régions comme au Bachkortostan, on trouvera dans ces raïons des grappes de villages oudmourtes homogènes. La situation linguistique y est-elle similaire? Quelle est la présence du russe dans ces régions? Jusqu'à quel point l'administration locale fonctionne en oudmourte ? Cela mériterait une étude ad hoc.

Est-il possible de reproduire une situation où le mode de fonctionnement communautaire marche aussi efficacement que dans le cas ici présenté ? Il est vrai qu'en Oudmourtie aussi, le système de gestion des troupeaux privés est identique, du moins dans quelques villages du sud (j'ai l'expérience de Karamas-Pel'ga, dans le raïon de Kijasovo, en 2008). Dans le village de Kuzebaevo, le seul sur le territoire de la 
République à avoir préservé les rituels oudmourtes, les mécanismes communautaires semblent être en place. Bien sûr, le poids des villes, de l'industrie, se fait plus fortement sentir, et elles viennent soutenir d'autres valeurs que celles qui dominent dans la vie rurale oudmourte. Surtout, en Oudmourtie, ce sont les villes, c'est la capitale, Iževsk, qui donnent le la. Même si, dans certaines campagnes, l'oudmourte garde toute sa vivacité, ce ne sont pas elles qui servent de modèle (cf. Nikitina 2013). Au Bachkortostan, il n'y a pas de ville pour jouer ce rôle. À Ufa, les Oudmourtes sont quantité négligeable. Les villageois ne vont pas aller chercher de modèles ailleurs, le modèle urbain est pour eux inexistant et non pertinent. C'est là une différence considérable.

Enfin, le bouclier tataro-bachkir n'existe pas en Oudmourtie. On peut identifier quelque chose de vaguement similaire en Oudmourtie du Sud: ce n'est pas un hasard si cette région, plus exposée que le reste de l'Oudmourtie aux influences tatares, est l'une de celles où le sens de l'identité nationale oudmourte est le plus prégnant, celle qui a donné naissance au plus grand nombre d'intellectuels oudmourtophones, au plus grand nombre de novateurs dans les années 1920. Mais cette influence, due à la proximité et à l'interpénétration territoriale des populations, n'empêche pas la présence russe à tous les niveaux des instances de pouvoir. Ce n'est pas un bouclier proprement dit.

Il y a pourtant, à mon sens, un message que les Oudmourtes du Bachkortostan peuvent transmettre à leurs compatriotes d'Oudmourtie, et c'est un encouragement. Il est aujourd'hui possible, en Russie, de rester soi-même, et que cela se passe de manière naturelle. L'oudmourte peut réellement servir, à tous les niveaux d'une société, de langue de communication et de pensée. Si cela ne marche pas de la sorte, la faute n'en est pas à l'oudmourte, mais à ceux qui ne veulent pas, ou ne savent pas, ou n'osent pas l'utiliser et en extraire toutes les possibilités. Les Oudmourtes d'outre-Kama sont bien placés pour encourager les Oudmourtes d'Oudmourtie à se prendre en main, à oser, à ne pas être timorés : ils vivent bien dans le même pays, et ils montrent qu'un modèle différent est possible. J'aimerais appeler les Oudmourtes d'Oudmourtie à regarder en direction de ces diasporas, à prendre modèle sur elles, car leur manière naturelle d'être peut leur donner des forces pour faire face aux obstacles qu'ils rencontrent ${ }^{17}$.

\section{BIBLIOGRAPHIE}

ARZAMAZOV Aleksej 2013, « Heurs et malheurs des langues finno-ougriennes de Russie », Études finno-ougriennes, $\mathrm{n}^{\circ}$ 45, Paris : L'Harmattan-ADEFO, p. 11-20.

GRANT Bruce, 1995, In the Soviet house of culture. A century of Perestroikas, Princeton University press. GREY Patty, 2000, The Predicament of Chukotka's Indigenous Movement: Post-Soviet Activism in the Russian Far North, Cambridge University Press.

JAMURZINA Ljudmila, 2010, "Conscience ethnique et représentations religieuses chez les Maris », Études finno-ougriennes, $\mathrm{n}^{\circ}$ 42, Paris : L'Harmattan-ADEFO, p. 83-102. 
JAMURZINA Ljudmila, 2013, « L'origine des Maris orientaux », Les Maris, un peuple finno-ougrien de Russie centrale, Paris : L'Harmattan-ADEFO, p. 111-126

KING Alexander D., 2011, Living with Koryak traditions. Playing with culture in Siberia, University of Nebraska Press.

MINNIYAKHMETOVA Tatiana, 1995, "Eating of beestings as an original calendar rite of the Bashkirian Udmurts”, Folk Belief Today, Tartu 1995.

MIROPIEV 1908 = МИРОПИЕВ М., «РУССКО-ИНОРОРДЧЕСКИЕ ШКОЛЫ СУСТЕМЫ Н.И. ИЛЬМИНСКОГО», ЖУРНАЛ МИНИСТЕРСТВА НАРОДНОГО ПРОСВЕЩЕНИЯ, 1, ЧАСТЬ ХІІІ, НОВАЯ СЕРИЯ, С. 183-210.

NIKITINA 2011 = НИКИТИНА ГАЛИНА, «ОБЩИЕ СВЕДЕНИЯ О ЗАКАМСКИХ УДМУРТОВ», Authentic Geography. ЗАКАМСКИЕ УДМУРТЫ, АЛЬМАНАХ 3, ИЖЕВСК 2011, С. 5-7

NIKITINA Galina, 2013, «Qui est responsable de la préservation des langues minoritaires ? Le cas de la langue oudmourte ", Études finno-ougriennes, $\mathrm{n}^{\circ}$ 45, Paris : L'Harmattan-ADEFO, p. 89-108.

PIKA Alexander, 1999, Neotraditionalism in the Russian North: Indigenous People and the Legacy of Perestroika, Edmonton: Canadian Circumpolar Institute Press.

RETHMAnN Petra, 2001, Tundra Passages: History and Gender in the Russian Far East, University Park, PA., Pennsylvania University Press,

RUETER Jack, 2013, “The Erzya language. Where is it spoken?” Études finno-ougriennes, $\mathrm{n}^{\circ} 45$, Paris : L'Harmattan-ADEFO, p. 21-40.

SADIKOV 2008 = САДИКОВ РАНУС РАФИКОВИЧ, ТРАДИЦИОННЫЕ РЕЛИГИОЗНЫЕ ВЕРОВАНИЯ И ОБРЯДНОСТЬ ЗАКАМСКИХ УДМУРТОВ (ИСТОРИЯ И СОВРЕМЕННЫЕ ТЕНДЕНЦИИ РАЗВИТИЯ), УФА: ЦЕНТР ЭТНОЛОГИЧЕСКИХ ИССЛЕДОВАНИЙ УНЦ РАН.

SADIKOV 2010 = САДИКОВ РАНУС РАФИКОВИЧ, „ЭЛЕН ВОСЬ - «МОЛЕНИЕ СТРАНОЙ» ЖИВАЯ ДРЕВНОСТЬ НА ПРОСТОРАХ БАШКИРИИ“ ВОРДСКЕМ КЫЛ, № 7, С. 34-36.

SADIKOV 2011 = САДИКОВ РАНУС РАФИКОВИЧ, «МУСУЛЬМАНЕ И ЯЗЫЧНИКИ: ПРОЦЕССЫ МЕЖКОНФЕССИОНАЛЬНЫХ ВЗАИМООТНОШЕНИЙ В СРЕДЕ ЗАКАМСКИХ УДМУРТОВ», ВЕСТНИК ВЭГУNo5(55), С. 89-94.

SADIKOV 2012 = САДИКОВ РАНУС РАФИКОВИЧ, РЕЛИГИОЗНЫЕ ВЕРОВАНИЯ И ОБРЯДНОСТЬ ЗАКАМСКИХ УДМУРТОВ (СОХРАНЕНИЕ И ПРЕЕМСТВЕННОСТЬ ТРАДИЦИИ).

SADIKOV, MINNIJAHMETOVA 2012 = САДИКОВ Р.Р., МИННИЯХМЕТОВА Т.Г., «ЗАРУБЕЖНЫЕ ИССЛЕДОВАТЕЛИ ЭТНОГРАФИИ, ФОЛЬКЛОРА И ЯЗЫКА ЗАКАМСКИХ УДМУРТОВ: ИСТОРИОГРАФИЧЕСКИЙ ОЧЕРК», ЕЖЕГОДНИК ФИННО-УГОРСКИХ ИССЛЕДОВАНИЙ, № 4. С. 49-62.

ŠEDA-ZORINA 2004 = ШЕДА-ЗОРИНА ИРИНА, «ЗАКАМСКИЕ УДМУРТЫ СЕГОДНЯ» АКТУАЛЬНОЕ НАЦИОНАЛЬНО-КУЛЬТУРНОЕ ОБОЗРЕНИЕ ВЫПУСК № 9, http://www.an-tat.ru/ob-assamblee/libraryassembly/magazine-anko/2330/2555/.

TOUlouze Eva, 2012, « Périple spirituel chez les Oudmourtes avec quelques portraits », Études finno-ougriennes, $n^{\circ} 43$, Paris : L'Harmattan-ADEFO, p. 250-264.

TOULouze Eva, “Fixity and movement in Western Siberia: When oil workers', natives' and reindeer's paths cross", in (Kannike Anu, ed.) Out and in between: Dynamics of cultural borders. Approaches to Culture Theory, nr. 5, Tartu : University of Tartu Press (à paraître). 
VENTSEL Aimar, 2005, “The State's indigenous policy, the centrally planned cultural Revival and Native Strategies of Survival", The Northern Peoples and States: changing relationships. Studies in Folk Culture, V, Tartu 2005, p. 164-183.

\section{NOTES}

1. Travaux effectués dans le raïon de Tatyšly avec Liivo Niglas, en vue de filmer et d'analyser des cérémonies animistes. Ce terrain a été réalisé dans le cadre de recherches financées par l'Union européenne par l'intermédiaire des Fonds européens de développement régional (Centre d'excellence CECT).

2. En russe : ЗАКАМСКИЕ УДМУРТЫ, terme utilisé pour la première fois en 1892 par N. I. Tezjakov (Sadikov 2012, p. 27)

3. Ainsi s'est exprimé le responsable du raïon, Rušam Garaev, un Tatar, lors de la journée du village d'Utar-Elga dans son discours introductif (le 15 juillet 2013, intervention filmée).

4. C'est ainsi que les Oudmourtes appellent familièrement ce village, "nouveau village». Officiellement, en oudmourte, son nom est Vil' Tatyšly.

5. Et peut-être aussi ailleurs.

6. En russe : НАЦИОНАЛЬНО-КУЛЬТУРНЫЙ ЦЕНТР УДМУРТОВ БАШКОРТОСТАНА.

7. Le même phénomène a eu lieu chez les Maris du Bachkortostan, et en général, d'outre-Kama (Jamurzina 2010).

8. Ce chiffre, comme les suivants, reprend les résultats officiels du recensement de 2010, http:// bashstat.gks.ru/wps/wcm/connect/rosstat_ts/bashstat/resources/

2f055a804e303140ba45fe3bf8d20d64/

ЧИСЛЕННОСТЬ+НАСЕЛЕНИЯ+ПО+НАСЕЛЕННЫМ+ПУНКТАМ+РЕСПУБЛИКИ+БАШКОРТОСТАН.pdf

9. Vös'as', nom désignant les prêtres qui animent les rituels et les sacrifices.

10. Mot turcique pour désigner la fête de fin des travaux de printemps. En juin, cette fête est organisée à tous les niveaux : village, kolkhoze, raïon, République. C'est l'occasion de remettre des primes pour les meilleurs travailleurs ou pour les meilleurs kolkhozes, de faire des discours, un spectacle, des jeux.

11. Chiffres du recensement de 2010.

12. De ce point de vue, les statistiques sont trompeuses. Bien que le recensement (2010) révèle un nombre de considérable de villages bachkirs dans le raïon de Tatyšly, sur place ces villages et leurs ressortissants sont considérés comme Tatars. Il s'agit clairement d'anciens Bachkirs tatarisés, qui ont le tatar comme langue maternelle. De manière générale, sauf dans les zones oudmourtes, le tatar est la langue dominante dans la communication ainsi que dans les prises de parole officielles.

13. http://www.webcitation.org/6EzC0FMGq, consulté le 24 juillet 2013.

14. J'ai développé la question du sens de ces notions dans un article récent (Toulouze, à paraître). Voir aussi Pika 1999, Grey 2000, Rethmann 2000, Grant 1995, Ventsel 2005, King 2011.

15. L'analyse qui précède s'appuie sur mes terrains et les nombreux entretiens que j'ai eu avec des autochtones de la région de la Volga. J'ai moi-même été témoin de manifestations d'hostilité et de mépris à l'égard de la langue oudmourte dans les transports en commun à Iževsk entre 1994 et 2004 .

16. http://shaer.ru/content/blogcategory/19/33/ (consulté le 25 juillet 2013).

17. Il leur suffirait de regarder dans leur société tous ceux qui, issus de contrées plus orientales, sont actifs et donnent l'exemple. Je pense aux écrivains Pëtr Zaharov, Ul'fat Badretidnov et Ljuza Badretdinova, frère et sœur, ou au linguiste Rif Nasibullin, qui œuvrent en Oudmourtie. 


\section{RÉSUMÉS}

Cet article représente une étude de cas, chez les Oudmourtes sis dans le nord du Bachkortostan. Cette diaspora, qui s'est formée entre le $\mathrm{XVI}^{\mathrm{e}}$ et le XVIII ${ }^{\mathrm{e}}$ siècle, a maintenu l'usage de la langue vernaculaire à un niveau tout à fait remarquable. Après avoir présenté rapidement ces communautés, je me concentrerai sur la situation linguistique, non seulement sur la base des statistiques les plus récentes, mais aussi sur la base des expériences sur le terrain. Enfin, j'analyserai les conditions qui ont permis cette réussite et je m'interrogerai sur comment les Oudmourtes d'Oudmourtie pourraient s'en inspirer.

This article is a case study by the Udmurt living in Northern Bashkortostan. This diaspora was formed between the 16th and the 18th century and has remarkably preserved the use of the vernacular. I first introduce the Udmurt communities and then concentrate on the linguistic situation not only on the basis of recent statistics but also through fieldwork experiences. I shall conclude by analysing the conditions that allowed this success story and reflect on the way Udmurtia Udmurt could be inspired by it.

Artiklis esitatakse case study Põhja-Baškortostani udmurtide näitel. See 16.-18. sajandil moodustatud diasporaa on üllatavalt hästi säilitanud oma keelekasutuse. Esiteks tutvustatakse Kaama jõe taguseid udmurdi kogukondi, seejärel keskendutakse keele olukorra kirjeldamisele mitte ainult hiljutise statistika vaid ka välitööde põhjal. Lõpetuseks analüüsitakse Baškortostani udmurtide keele säilimise tingimusi ning juureldakse, kuidas Udmurdimaa udmurdid võiksid sellest inspiratsiooni saada.

\section{INDEX}

Index géographique : Bal'zuga, Alga, Ariba, Bigineevo, Dubovka, Ivanovka, Juda, Majsk, Nižnebaltačevo, Ufa, Petropavlovka, Stary Kyzyl'yar, Tanypovka, Urazgyldy, Utar Elga, Verhnebaltačevo, Verkhnye Tatyshly, Vjazovka, Kueda, Kirga, Tatarstan (République), Kazan, Starokal'miyarovo, Alnaši, Debessy, Glazov, Iževsk, Karamas-Pel'ga, Kiassovo, Kuzebaevo, Malaya Purga, Šarkan, Oudmourtie (République)

Thèmes : linguistique

disciplines oudmourte

Keywords : Udmurt, Diaspora, Islam, traditional society, Bachkortostan, Alga, Aribash, Bal'zyuga, Bigineevo, Dubovka, Ivanovka, Yuda, Maysk, Nizhnebaltachevo, Ufa, Petropavlovka, Starokal'miyarovo, Stary Kyzyl'yar, Tanypovka, Urazgyldy, Utar Elga, Verkhnebaltachevo, Verkhnye Tatyshly, Vil' Tatyshly Vil'gurt (Novye Tatyshly), Vyazovka, Perm Oblast, Kueda, Kirga, Tatartan, Kazan, Udmurtia, Alnashi, Debessy, Glazov, Izhevsk, Karamas-Pel'ga, Kiassovo, Kuzebaevo, Malaya Purga, Sharkan, Bashkir, Estonian, Russians, Tatars

Mots-clés : diaspora, islam, société traditionnelle 